\title{
Bilateral and multiple mixed epithelial and stromal tumors of the kidney: A case report
}

\author{
RYO MINODA $^{1}$, TOSHIO TAKAGI ${ }^{1}$, NAOHIRO TODA ${ }^{1}$, HIROKO ITAGAKI $^{2}$, \\ TSUNENORI KONDO $^{1}$, HIDEKI ISHIDA ${ }^{1}$, YOJI NAGASHIMA ${ }^{2}$ and KAZUNARI TANABE ${ }^{1}$ \\ Departments of ${ }^{1}$ Urology and ${ }^{2}$ Surgical Pathology, Tokyo Women's Medical University, Tokyo 162-8666, Japan
}

Received March 9, 2017; Accepted September 28, 2017

DOI: $10.3892 / \mathrm{mco} .2017 .1444$

\begin{abstract}
Mixed epithelial and stromal tumor (MEST) of the kidney is a benign tumor occurring predominantly in older women. Histologically, MEST comprises tubuloglandular and cystic structures in an ovarian-like stroma. Bilateral and multiple cases are rare. We herein present a case of bilateral and multiple MEST in a 43-year-old woman. The patient presented with gross hematuria and was referred to a primary care physician. A computed tomography scan revealed 5 tumors in the right and 2 in the left kidney. Renal cell carcinoma was suspected, and left partial nephrectomy followed by right radical nephrectomy was planned. After the left partial nephrectomy, the histopathological diagnosis was MEST, which was consistent with the results obtained following needle biopsy. Therefore, the contralateral tumor was also suspected to be MEST, and needle biopsy was performed. The histopathological diagnosis was identical and the right kidney was spared. The patient is currently periodically monitored. As MEST is a benign tumor, preoperative diagnosis is crucial for avoiding overtreatment.
\end{abstract}

\section{Introduction}

Mixed epithelial and stromal tumor (MEST) is a biphasic renal tumor predominant in women, possessing epithelial (tubular glandular architecture) and stromal (similar to the ovarian stroma, expressing estrogen and progesterone receptors and CD10) elements, with only 10 diagnosed cases among 4,532 renal tumors between 1987 and 2005 as reported by the Cleveland Clinic (1). MEST is classified as a mixed

Correspondence to: Dr Ryo Minoda, Department of Urology, Tokyo Women's Medical University, 8-1 Kawada-cho, Shinjuku-ku, Tokyo 162-8666, Japan

E-mail: ryomnoda@gmail.com

Abbreviations: MEST, mixed epithelial and stromal tumor; CT, computed tomography; RCC, renal cell carcinoma

Key words: mixed epithelial and stromal tumor, bilateral, multiple, kidney mesenchymal and epithelial tumor in the current World Health Organization classification (2). Tumorigenesis is likely hormonally determined. These tumors are mostly benign, but malignant transformation of the stromal element has also been reported (1).

Computed tomography (CT) shows several variants, including solid enhancing components or cystic tumors, such as Bosniak III or IV. This suggests that differential diagnosis from cystic renal cell carcinoma (RCC) and/or plain RCC may be challenging. Renal MEST is usually resected based on a preoperative diagnosis of RCC, which may lead to surgical overtreatment.

Bilateral and multiple cases are rarely reported (1). We herein present a case with bilateral and multiple MESTs, in which overtreatment was avoided.

\section{Case report}

A 43-year-old Japanese woman consulted a primary care physician for gross hematuria. The patient's medical and family history was unremarkable. Urinalysis revealed mild occult blood reaction 1+ and red blood cells (RBCs) 30-49/high-power field. The serum creatinine level and estimated glomerular filtration rate were $0.64 \mathrm{mg} / \mathrm{dl}$ and $79.9 \mathrm{ml} / \mathrm{min} / 1.73 \mathrm{~m}^{2}$, respectively. On MAG3 renal scintigraphy, the split renal function was 41 and 59\% in the right and left kidneys, respectively. On abdominal CT, 2 tumors (73 and $28 \mathrm{~mm}$ in diameter) were identified in the left kidney, and 5 tumors $(15-50 \mathrm{~mm}$ in diameter) were identified in the right kidney. The tumors were heterogeneous on CT, appearing as enhanced masses including cysts with irregular septa, suggesting Bosniak IV cystic RCC; solid masses were observed, which were enhanced in the corticomedullary phase and washed out in the delayed phase (Fig. 1). Genetic testing excluded Von Hippel-Lindau disease. Therapy was planned based on the preoperative diagnosis of RCC. Preserving the right kidney appeared to be difficult due to the presence of 5 large tumors. Therefore, left partial nephrectomy followed by right radical nephrectomy was planned. Following left nephrectomy, the resected tumors were pathologically diagnosed as MESTs, without malignant characteristics. Accordingly, the right kidney tumors were also suspected to be MESTs and a CT-guided biopsy confirmed this diagnosis; thus, the right kidney was spared. The patient is currently periodically followed-up with CT every 4-6 months. 
Grossly, the partially resected left kidney contained two well-demarcated, brownish tumors. The cut surface of the tumors was mostly solid and firm, with occasional cystic spaces (Fig. 2).

Histologically, the tumors were well demarcated from the surrounding renal parenchyma, without a pseudocapsule. The tumors predominantly comprised a stromal element, with bland spindle cells. The stroma exhibited various-sized tubuloglandular and cystic formations lined by columnar epithelia, without atypia. The stromal cells were spindle-shaped, with scanty cytoplasm and without nuclear atypia. Immunohistochemically, the stroma cells were positive for estrogen receptor (ER), progesterone receptor (PR), $\alpha$-smooth muscle actin (SMA) and vimentin (Fig. 3). The pathological and immunohistochemical findings were compatible with the diagnosis of MEST. There were only few structurally abnormal cells and the mitotic count was low, suggesting benign MEST.

The needle biopsy specimen from the contralateral kidney also confirmed the diagnosis of MEST; thus, the right kidney was spared.

\section{Discussion}

MEST is a biphasic tumor comprising epithelial and stromal components, and is classified as a mixed mesenchymal and epithelial tumor in the current World Health Organization classification (2). Of the 4,532 renal tumors, $10(0.2 \%)$ were diagnosed as MEST between 1987 and 2005 at the Cleveland Clinic (1). The mean patient age was 46 years, with a male:female ratio of 1:6 $(3,4)$. In men, MEST tends to occur following endocrine treatment for prostate cancer. Bilateral cases are rare, with the first bilateral MEST reported in 2008 by Sangoi et al (5).

The present case is rare, and presented with gross hematuria. Lane et al reported that $67 \%$ of the patients presented with hematuria, flank pain and a palpable mass (1). Recent studies reported the most common characteristics of MEST to be a flank mass (31.8\%) and gross hematuria (27.3\%) (4). A total of $25 \%$ of MESTs are incidentally discovered, and they comprise $0.20-0.28 \%$ of all renal tumors (6). The tumors are generally sized $>5 \mathrm{~cm}$ (mean, $12.3 \mathrm{~cm}$ ) (4). The patient presented herein also had large tumors (2 tumors with diameters of 73 and $28 \mathrm{~mm}$ in the left kidney; and 5 tumors sized $10-51 \mathrm{~mm}$ in the right kidney), resulting in gross hematuria.

Radiologically, MEST includes various proportions of solid and cystic components. Lane et al reported that 7/10 (70\%) MESTs had solid enhancing components, including 3 Bosniak IV lesions and 4 solid enhancing lesions (1). Additionally, the radiological characteristics of MEST include thin, hair-like septae, curvilinear calcifications an and irregular surface (1). These characteristics were consistent with those observed in the present case. The left kidney tumors exhibited solid components that were enhanced in the early phase and washed out in the delayed phase, which caused difficulty in distinguishing them from clear-cell RCCs. However, the right kidney tumors displayed mixed characteristics, including solid masses and cystic lesions with enhancing solid components, suggesting Bosniak IV RCC. Thus, the preoperative diagnosis of MEST based solely on radiological characteristics is likely problematic.

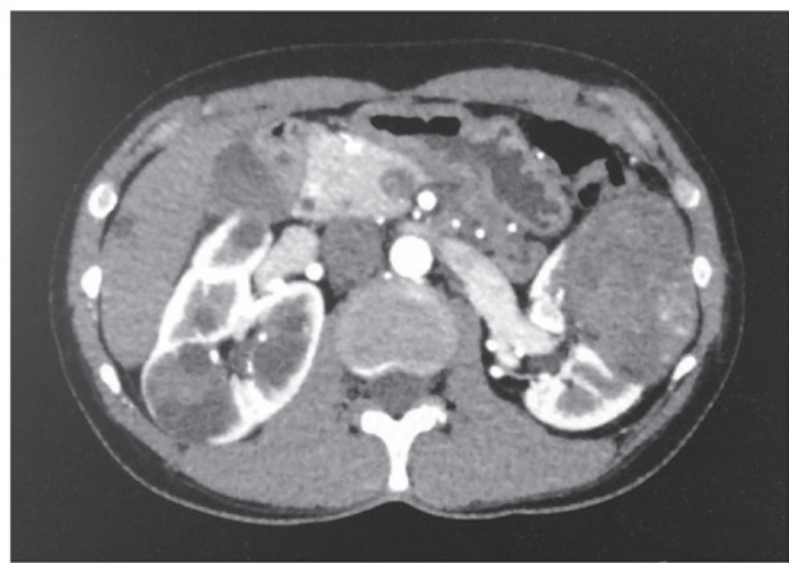

Figure 1. Enhanced computed tomography scan showing 2 solid tumors with a diameter of 73 and $28 \mathrm{~mm}$ in the left kidney, enhanced in the early phase and washed out in the delayed phase, and 5 tumors (1 solid and 4 cystic with small enhancing areas) in the right kidney, suggestive of Bosniak IV renal cell carcinoma.

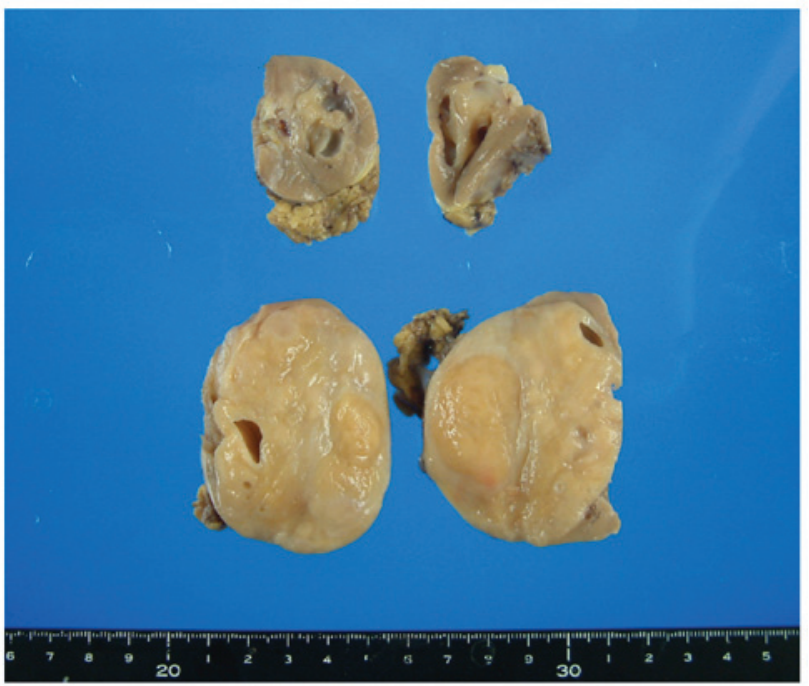

Figure 2. Gross findings of the partially resected left kidney. The tumor was well-demarcated, mostly solid with occasional cystic spaces, and firm in consistency.

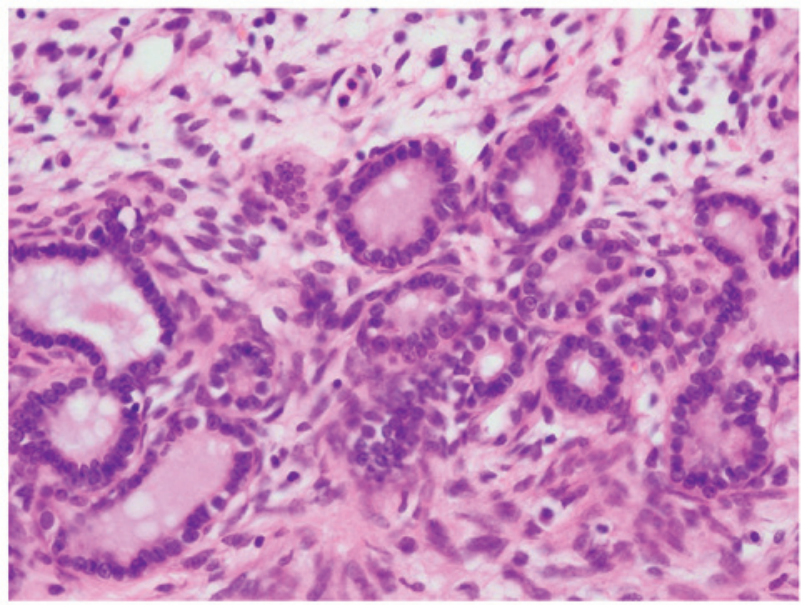

Figure 3. The microscopic findings included various-sized tubulocystic formations embedded in an ovarian-like stroma. (hematoxylin and eosin staining; original magnification, $\mathrm{x} 40$ ). 

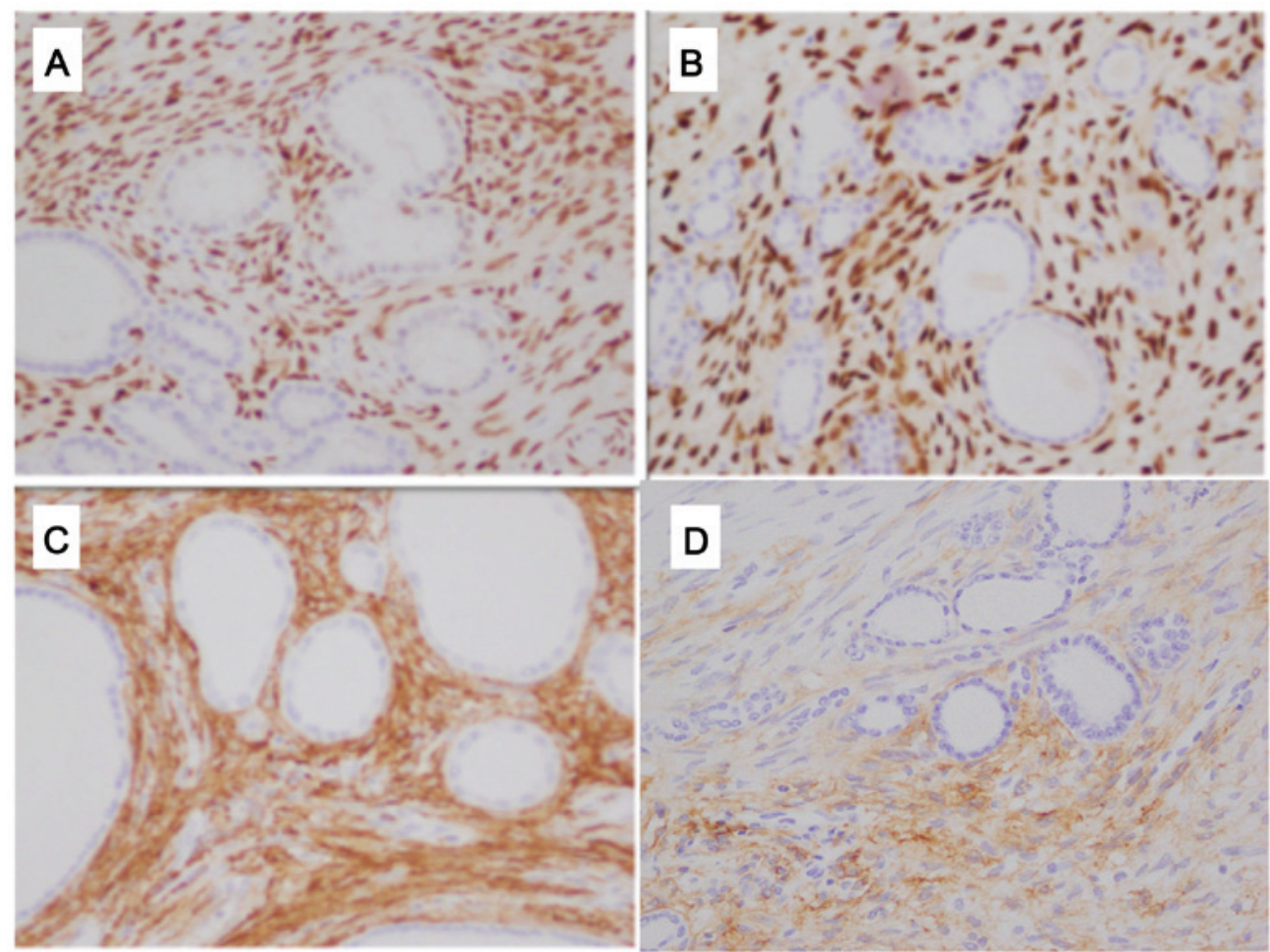

Figure 4. The immunohistochemical examination revealed positivity of the stromal component for (A) estrogen receptor, (B) progesterone receptor, (C) $\alpha$-smooth muscle actin and (D) vimentin. Original magnification, $x 40$.

Grossly, the proportion of cystic to solid component varies. In cases with a dominant cystic architecture, cystic nephroma may be the suspected diagnosis. The microscopic characteristics of MEST are epithelial cells arranged in a tubulocystic pattern in a background of stroma with bland spindle cells $(4,7)$. Immunohistochemically, MEST stains positive for epithelial components with antibodies to cytokeratin (CK), particularly CK7. Stromal components express vimentin, SMA, caldesmon and desmin, whereas expression of CD10, calretinin, inhibin, ER and PR may also be resent (4). ER and PR were positive in 4 and 2 of 4 cases, respectively (1). The tumors in the present case were positive for ER, PR, $\alpha$-SMA and vimentin.

MESTs are generally benign, but rare cases of malignant transformation have been reported $(8,9)$. Some cases also exhibit an aggressive course, with tumor recurrence or disease-related death $(8,9)$. Lane et al (1) reported that 1 of $10(10 \%)$ MESTs had a malignant sarcomatous component. In the present case, the 2 left kidney tumors were completely removed by partial nephrectomy and the 5 right kidney tumors remained untreated. These 5 tumors included 4 cystic tumors with small enhancing solid components and 1 solid tumor. The solid tumor was pathologically diagnosed as benign MEST following CT-guided needle biopsy. In the majority of previous reports, MEST was surgically removed, as RCC was suspected and preoperative radiological diagnosis of MEST is difficult. Therefore, active surveillance of unresected MESTs has not been previously reported. However, there is a possibility that the remaining tumors are malignant, although the solid parts in these cystic lesions are scarce, so the possibility of a rapidly growing malignant tumor is low. Although right kidney tumor removal was not performed, the patient undergoes periodical
CT check-ups every 4-6 months to monitor the malignant potential in the remaining tumors.

\section{References}

1. Lane BR, Campbell SC, Remer EM, Fergany AF, Williams SB, Novick AC, Weight CJ, Magi-Galluzzi C and Zhou M: Adult cystic nephroma and mixed epithelial and stromal tumor of the kidney: Clinical, radiographic, and pathologic characteristics. Urology 71: 1142-1148, 2008.

2. Eble JN, Sauter G, Epstein JI and Sesterhenn IA: World Health Organization Classification of Tumors. Pathology and Genetics of Tumours of the Urinary System and Male Genital Organs. IARC Press, Lyon, 2004

3. Montironi R, Mazzucchelli R, Lopez-Beltran A, Martignoni G, Cheng L, Montorsi F and Scarpelli M: Cystic nephroma and mixed epithelial and stromal tumour of the kidney: Opposite ends of the spectrum of the same entity? Eur Urol 54: 1237-1246, 2008.

4. Moslemi MK: Mixed epithelial and stromal tumor of the kidney or adult mesoblastic nephroma: An update. Urol J 7: 141-147, 2010.

5. Sangoi AR and Higgins JP: Bilateral mixed epithelial stromal tumor in an end-stage renal disease patient: The first case report. Hum Pathol 39: 142-146, 2008.

6. Terao H, Makiyama K, Yanagisawa M, Miyake M, Sano F, Kita K, Murakami T, Nakaigawa N, Ogawa T, Uemura H, et al: Mixed epithelial and stromal tumor of kidney: A case report. Hinyokika Kiyo 55: 495-498, 2009 (In Japanese).

7. Truong LD, Williams R, Ngo T, Cawood C, Chevez-Barrios P, Awalt HL, Brown RW, Younes M and Ro JY: Adult mesoblastic nephroma: Expansion of the morphologic spectrum and review of literature. Am J Surg Pathol 22: 827-839, 1998.

8. Suzuki T, Hiragata S, Hosaka K, Oyama T, Kuroda N, Hes O and Michal M: Malignant mixed epithelial and stromal tumor of the kidney: Report of the first male case. Int J Urol 20: 448-450, 2013.

9. Adsay NV, Eble J, Srigley JR, Jones EC and Grignon DJ: Mixed epithelial and stromal tumor of the kidney. Am J Surg Pathol 24: 958-970, 2000. 